\title{
Assessing the clinical utility of genetic risk scores for targeted cancer screening
}

Carly A. Conran ${ }^{1 *} \mathbb{0}$, Zhuqing Shi ${ }^{2}$, William Kyle Resurreccion ${ }^{2}$, Rong $\mathrm{Na}^{3}$, Brian T. Helfand ${ }^{2}$, Elena Genova ${ }^{2}$, Siqun Lilly Zheng ${ }^{2}$, Charles B. Brendler ${ }^{2}$ and Jianfeng $\mathrm{Xu}^{2}$

\begin{abstract}
Background: Genome-wide association studies have identified thousands of disease-associated single nucleotide polymorphisms (SNPs). A subset of these SNPs may be additively combined to generate genetic risk scores (GRSs) that confer risk for a specific disease. Although the clinical validity of GRSs to predict risk of specific diseases has been well established, there is still a great need to determine their clinical utility by applying GRSs in primary care for cancer risk assessment and targeted intervention.
\end{abstract}

Methods: This clinical study involved 281 primary care patients without a personal history of breast, prostate or colorectal cancer who were 40-70 years old. DNA was obtained from a pre-existing biobank at NorthShore University HealthSystem. GRSs for colorectal cancer and breast or prostate cancer were calculated and shared with participants through their primary care provider. Additional data was gathered using questionnaires as well as electronic medical record information. A t-test or Chi-square test was applied for comparison of demographic and key clinical variables among different groups.

Results: The median age of the 281 participants was 58 years and the majority were female (66.6\%). One hundred one (36.9\%) participants received 2 low risk scores, 99 (35.2\%) received 1 low risk and 1 average risk score, 37 (13.2\%) received 1 low risk and 1 high risk score, 23 (8.2\%) received 2 average risk scores, 21 (7.5\%) received 1 average risk and 1 high risk score, and no one received 2 high risk scores. Before receiving GRSs, younger patients and women reported significantly more worry about risk of developing cancer. After receiving GRSs, those who received at least one high GRS reported significantly more worry about developing cancer. There were no significant differences found between gender, age, or GRS with regards to participants' reported optimism about their future health neither before nor after receiving GRS results.

Conclusions: Genetic risk scores that quantify an individual's risk of developing breast, prostate and colorectal cancers as compared with a race-defined population average risk have potential clinical utility as a tool for risk stratification and to guide cancer screening in a primary care setting.

Keywords: Genetic risk score, Single nucleotide polymorphism, Translational genomics, Cancer screening, Breast cancer, Prostate cancer, Colorectal cancer

*Correspondence: carlyconran@gmail.com

${ }^{1}$ University of Illinois College of Medicine, Chicago, IL, USA

Full list of author information is available at the end of the article

\section{Background}

As genome-wide association studies (GWASs) have become increasingly important since their conception in 2008, scientists have made substantial progress in the field of genetic epidemiology. Specifically, thousands of disease-associated single nucleotide polymorphisms (SNPs) have been identified. Many of these SNPs may be

(c) The Author(s) 2021. This article is licensed under a Creative Commons Attribution 4.0 International License, which permits use, sharing, adaptation, distribution and reproduction in any medium or format, as long as you give appropriate credit to the original author(s) and the source, provide a link to the Creative Commons licence, and indicate if changes were made. The images or other third party material in this article are included in the article's Creative Commons licence, unless indicated otherwise in a credit line to the material. If material is not included in the article's Creative Commons licence and your intended use is not permitted by statutory regulation or exceeds the permitted use, you will need to obtain permission directly from the copyright holder. To view a copy of this licence, visit http://creativeco mmons.org/licenses/by/4.0/. The Creative Commons Public Domain Dedication waiver (http://creativecommons.org/publicdomain/ zero/1.0/) applies to the data made available in this article, unless otherwise stated in a credit line to the data. 
additively combined to generate polygenic risk scores, or genetic risk scores (GRSs), that confer risk for a specific disease. Although the clinical validity of GRSs to predict risk of specific diseases has been well established in seminal publications, no healthcare system, to our knowledge, has assessed their clinical utility by applying GRSs in a primary care setting for cancer risk assessment and targeted intervention.

At present, the screening recommendations for colorectal, breast, and prostate cancers used in primary care are based on risk assessment models that incorporate age, family history (FH), and varying clinical markers [1-4]. In the era of personalized medicine, however, it has been demonstrated that these risk assessment models can be improved by including genetic information to more accurately estimate an individual's risk of developing these specific cancers [5-9]. There is a need for this more objective and personalized information, as screening recommendations now rely on divergent guidelines and possibly imprecise, incomplete or unknown $\mathrm{FH}$ information. Furthermore, these currently available models are useful only for the minority of individuals who have a positive $\mathrm{FH}$; as most individuals who develop cancer, especially at a young age, do not have a known FH of the disease [9-11].

Through the present clinical trial, GRSs were used clinically as part of a multivariate model that included $\mathrm{FH}$ of disease as well as other clinical risk factors, to stratify patients based on their individual genetic risk of developing a disease. Investigators, in consultation with surgical oncology specialists, developed a unique genetic test to identify SNPs as well as implementation models (Additional file 1: Appendix A) to guide more personalized risk stratification and screening for colorectal, breast, and prostate cancers in a primary care setting. However, it was important to assess whether increased worry among patients about the possibility of developing cancer would outweigh the benefits of this gained knowledge.

\section{Methods}

\section{Design and study population}

This clinical study involved primary care patients without a personal history of breast, prostate or colorectal cancers who were 40-70 years old at enrollment. All participants were recruited from an existing IRB-approved genomic study, called the Genomic Health Initiative at NorthShore University HealthSystem, in which DNA samples had already been collected and stored. Participants were re-contacted and provided additional written informed consent for the current study.

The study also enrolled a second group: 59 primary care physicians (PCPs). Here, we will be discussing only the experiences of the 281 participants who were enrolled in the study. Briefly, the Genomic Health Initiative database was queried for individuals who met eligibility criteria. PCPs of the identified individuals were contacted for participation in the physician arm of the study. PCP involvement required watching educational videos about GRSs and confirming comfort with reporting results. Videos included basic genetic information about DNA and risk-associated SNPs, a cancer risk assessment overview involving family history and GRSs, and modified cancer screening guidelines that incorporate GRSs into standard of care for all three cancers. Participants were not contacted until their PCP approved our recruitment of their patients. Ultimately, the number of participants enrolled under individual PCPs ranged from 1 to 21 participants.

Of note, certain patient populations were excluded, as there is insufficient evidence for calculating GRSs in people of certain descents. Examples include: mixed races, West Asians (e.g. Middle Eastern), Native Americans, and Hispanics. Participants with a known BRCA1 or $B R C A 2$ mutation were also excluded, as their level of risk for developing breast or prostate cancers is likely higher than the risk conferred by a high GRS.

The study protocol was approved by the NorthShore University HealthSystem Institutional Review Board in Evanston, IL. All participants provided written informed consent.

\section{Intervention}

An aliquot of each participant's DNA was extracted from the Genomic Health Initiative biorepository and sent to Counsyl, Inc. in San Francisco, CA. Counsyl is a private genomics company that was contracted by NorthShore to sequence patients' de-identified DNA samples for 299 cancer risk-associated SNPs (Additional file 2: Table 2). A custom panel was developed with templates for the SNP regions.

After DNA samples were collected and analyzed, two GRSs were generated for each participant: a breast cancer GRS and colorectal cancer GRS for each female, and a prostate cancer GRS and colorectal cancer GRS for each male. GRSs were calculated using the equations $\mathrm{GRS}=\prod_{\mathrm{i}=1}^{\mathrm{n}} \frac{\mathrm{OR}_{\mathrm{i}}^{\mathrm{S}_{1}}}{\mathrm{~W}_{\mathrm{i}}}$ and $\mathrm{W}_{\mathrm{i}}=\mathrm{f}_{\mathrm{i}}^{2} \mathrm{OR}_{\mathrm{i}}^{2}+2 \mathrm{f}_{\mathrm{i}}\left(1-\mathrm{f}_{\mathrm{i}} \mathrm{OR}_{\mathrm{i}}+\left(1-\mathrm{f}_{\mathrm{i}}\right)^{2}\right.$, where $\mathrm{g}_{\mathrm{i}}$ represents the individual's SNP i genotype $(0,1$, or 2 risk alleles), $\mathrm{OR}_{\mathrm{i}}$ represents the allelic OR of SNP $\mathrm{i}$, and $f_{i}$ represents the risk allele frequency of SNP $i$ in the population.

Data was collected from two sources: questionnaires and participants' electronic medical records. Participants were asked to respond to three questionnaires timed as follows: before taking the GRS test (i.e. shortly after recruitment), immediately after receiving test results, and three months after results were returned. Questionnaires 
assessed participants' feelings about their health, specifically as it related to cancer risk, as well as their plans for breast, prostate and/or colorectal cancer screening. Questionnaires were sent to participants in the format of their choosing: via email using REDCap software, or via postal mail. The questionnaires used were similar to those previously used to collect data on worry about cancer risk and diagnosis, including the US Health Information National Trends Survey (US HINTS), Lerman's Cancer Worry Scale and the Cancer Worry Scale for Genetic Counseling [12-18]. Cancer screening-related health information was also collected throughout the follow up period of 18-42 months (data not reported).

\section{Assessment of genetic risk}

A GRS is an odds ratio value that confers one's risk of developing a specific disease relative to average population risk. For example, a woman with a breast cancer GRS equal to 2.0 indicates that she is twice as likely as the average woman to develop breast cancer in her lifetime ( 26\% likelihood vs 12.9\%) [19].

GRS values were reported to participants, as well as their category of risk. GRSs in this study were categorized into three groups: low risk, average risk or high risk. Values were categorized as follows, based on varying GRSs corresponding with relative risk and absolute lifetime risks from meta analyses and/or cohort studies: [20-26]

\begin{tabular}{llll}
\hline Cancer type & Low risk GRS & Average risk GRS & High risk GRS \\
\hline Breast & $<0.5$ to $<1.0$ & $1.0-1.4$ & 1.5 to $>3.0$ \\
Prostate & $<0.5$ to $<1.0$ & $1.0-1.6$ & 1.7 to $>3.0$ \\
Colorectal & $<0.5$ to $<1.0$ & $1.0-1.6$ & 1.7 to $>3.0$ \\
\hline
\end{tabular}

Of note, GRS values for any disease of $<0.5$ or $>3.0$ were reported as " $<0.5$ " or " $>3.0$," respectively (i.e. exact values for exceptionally low or high scores were not reported).

\section{Result reporting}

GRSs were shared with participants through their electronic medical record portal by their PCPs. PCPs signed a separate consent form to also participate in the study and watched educational videos about GRSs before returning any results. Suggested personalized genomicbased screening guidelines that incorporated GRSs were provided to PCPs for their consideration (Additional file 1: Appendix A).

\section{Statistical analysis}

A t-test or Chi-square test was applied for comparison of demographic and key clinical variables among different groups. P-values for continuous variables (e.g. age) are a p of linear regression, and the p-values for categorical variables (e.g. gender) are a $\mathrm{p}$ for the proportion trend test. All statistical analyses were performed using $\mathrm{R}$ v3.5.2 [27]. A two-tailed $P<0.05$ was considered statistically significant.

\section{Results}

\section{Patient characteristics}

The median age of the 281 participants was 58 years. There were more female participants (66.6\%). The majority of participants were Caucasian (95.0\%), with fewer African American (3.2\%) and East Asian (1.8\%) participants. No participants had a personal history of breast, prostate or colorectal cancer. Participants reported their $\mathrm{FH}$ of breast, prostate and colorectal cancers in first- and second-degree family members. Among all participants (both male and female), 35.2\% reported a FH of breast cancer, $25.2 \%$ reported a $\mathrm{FH}$ of prostate cancer, and $25.6 \%$ reported a FH of colorectal cancer (Table 1).

\section{Genetic risk scores and family history}

As each participant received 2 GRSs, they can be grouped by their risk category: 101 (36.9\%) participants received 2 "low risk" scores, 99 (35.2\%) received 1 "low risk" score and 1 "average risk" score, 37 (13.2\%) received 1 "low risk" score and 1 "high risk" score, 23 (8.2\%) received 2 "average risk" scores, 21 (7.5\%) received 1 "average risk" score and 1 "high risk" score, and no one received 2 "high

\section{Table 1 Demographics}

\begin{tabular}{ll}
\hline Sex & \\
Male & $94(33.45 \%)$ \\
Female & $187(66.55 \%)$ \\
Age & \\
$40-49$ & $56(19.93 \%)$ \\
$50-59$ & $94(33.45 \%)$ \\
$60-70$ & $131(46.62 \%)$ \\
Race & \\
Caucasian & $267(95.02 \%)$ \\
African American & $9(3.2 \%)$ \\
East Asian & $5(1.78 \%)$ \\
Family history & \\
Breast cancer & Missing: 13 \\
Male & $30(34.09 \%)$ \\
Female & $69(38.33 \%)$ \\
Prostate cancer & Missing: 12 \\
Male & $24(26.67 \%)$ \\
Female & $47(26.26 \%)$ \\
Colorectal cancer & Missing:8 \\
Male & $23(25.56 \%)$ \\
Female & $49(26.78 \%)$ \\
\hline
\end{tabular}


risk" scores (Fig. 1a). For each type of cancer, the majority of participants were found to be "low risk" based on GRS, with $62.0 \%$ of breast cancer, $66.0 \%$ of prostate cancer, and $56.9 \%$ of colorectal cancer GRSs being low risk (Fig. 1ac). Fewer GRSs for each cancer type were "high risk," with $11.8 \%$ of breast cancer, $20.2 \%$ of prostate cancer and $6.1 \%$ of colorectal cancer GRSs being high risk (Fig. 1a-c).

We also analyzed the combined risk of GRS with FH within individuals (Additional file 3: Supplemental Figure 1). Among the 180 women with FH information available in this study, $69(38.33 \%)$ reported a family history of breast cancer and $20(11.11 \%)$ had a high GRS for breast cancer. Seven (7) (3.89\%) women had both a family history and high GRS for breast cancer. Among the 90 men with FH information available, $24(26.67 \%)$ reported a family history of prostate cancer and 18 (20\%) had a high GRS for prostate cancer. Five (5) (5.56\%) men had both a family history and high GRS for prostate cancer. Among the 273 women and men with family history information available for colorectal cancer, 72 (26.37\%) reported a family history of colorectal cancer and 17 (6.23\%) had a high GRS for colorectal cancer. Five (5) (1.83\%) had both a family history and high GRS for colorectal cancer.

\section{Attitudes regarding GRS-related health}

Participants were asked to rate the degree to which they agreed with three statements both before and after receiving their GRS results. Response choices, in order of level of agreement, were: "not at all," "very little," "somewhat," "quite a bit," or "a great deal."
The three pre-result statements were: (1) I feel anxious when I think about getting my Genetic Risk Scores; (2) I worry about my risk of having cancer when I think about finding out my Genetic Risk Scores; and (3) I feel optimistic regarding my future health. The three corresponding statements that participants responded to after receiving their GRSs were: (1) I feel anxious knowing my Genetic Risk Scores; (2) I worry about my risk of developing cancer; and (3) I feel optimistic regarding my future health.

Before receiving GRSs, younger patients and women reported significantly more worry about risk of developing cancer (Fig. 2). The average age of participants who agreed "quite a bit" or "a great deal" with the statement regarding worrying about cancer was 52.1 years, whereas the average age of those responding "somewhat" was 56.4 years, and those responding "not at all" or "very little" was 59.3 years $(\mathrm{p}=1.83 \mathrm{E}-4)$. The proportion of women who indicated that they agreed "quite a bit" or "a great deal" to the same statement about worrying about cancer was significantly higher $(88.0 \%)$ than the proportion of women who stated "somewhat" $(72.7 \%)$, or "not at all" or "very little" $(61.5 \%)(\mathrm{p}=0.01)$.

After receiving GRSs, participants who had at least one high GRS reported agreeing significantly more strongly with the statement "I feel anxious knowing my Genetic Risk Scores" than those who had no high GRS (Fig. 2). Of people who responded "quite a bit" or "a great deal," $88.9 \%$ had at least one high GRS, whereas of those who responded "somewhat," $56 \%$ had at least one high GRS, and of those who responded "not at all"
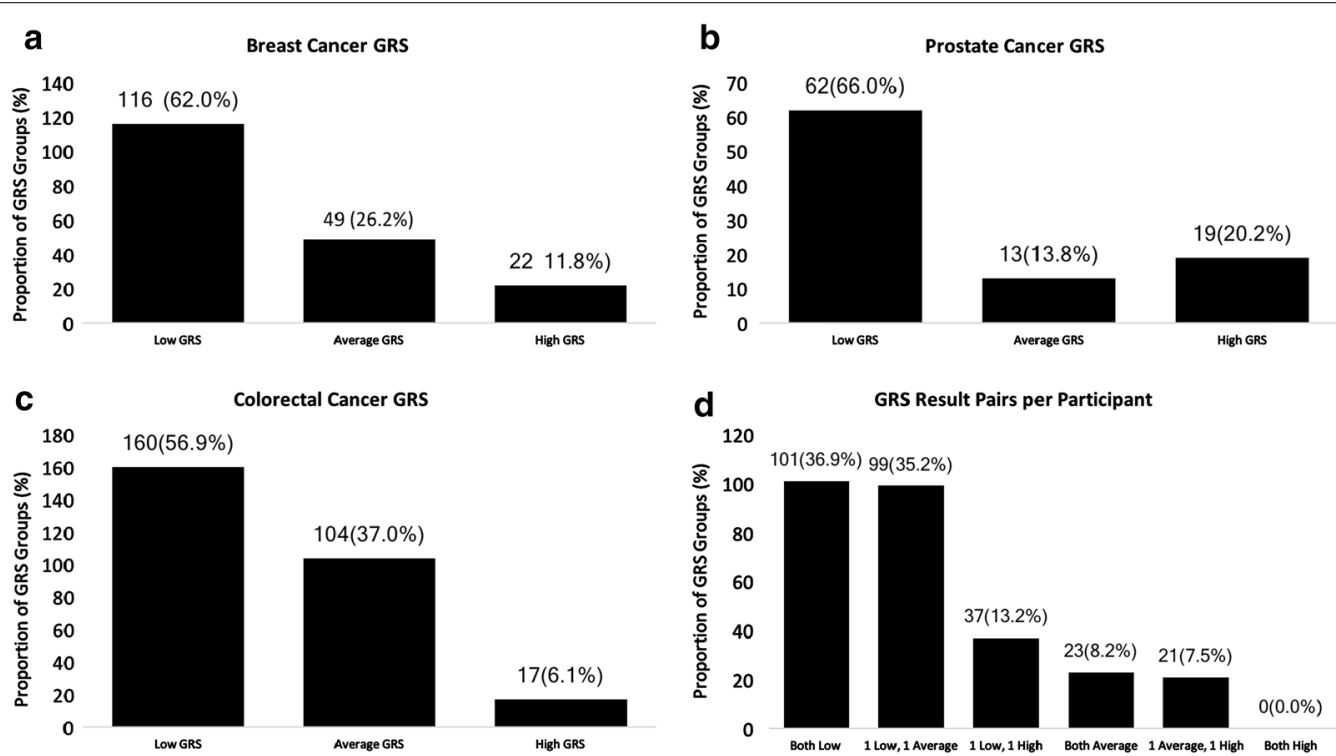

Fig. 1 Genetic risk score results 

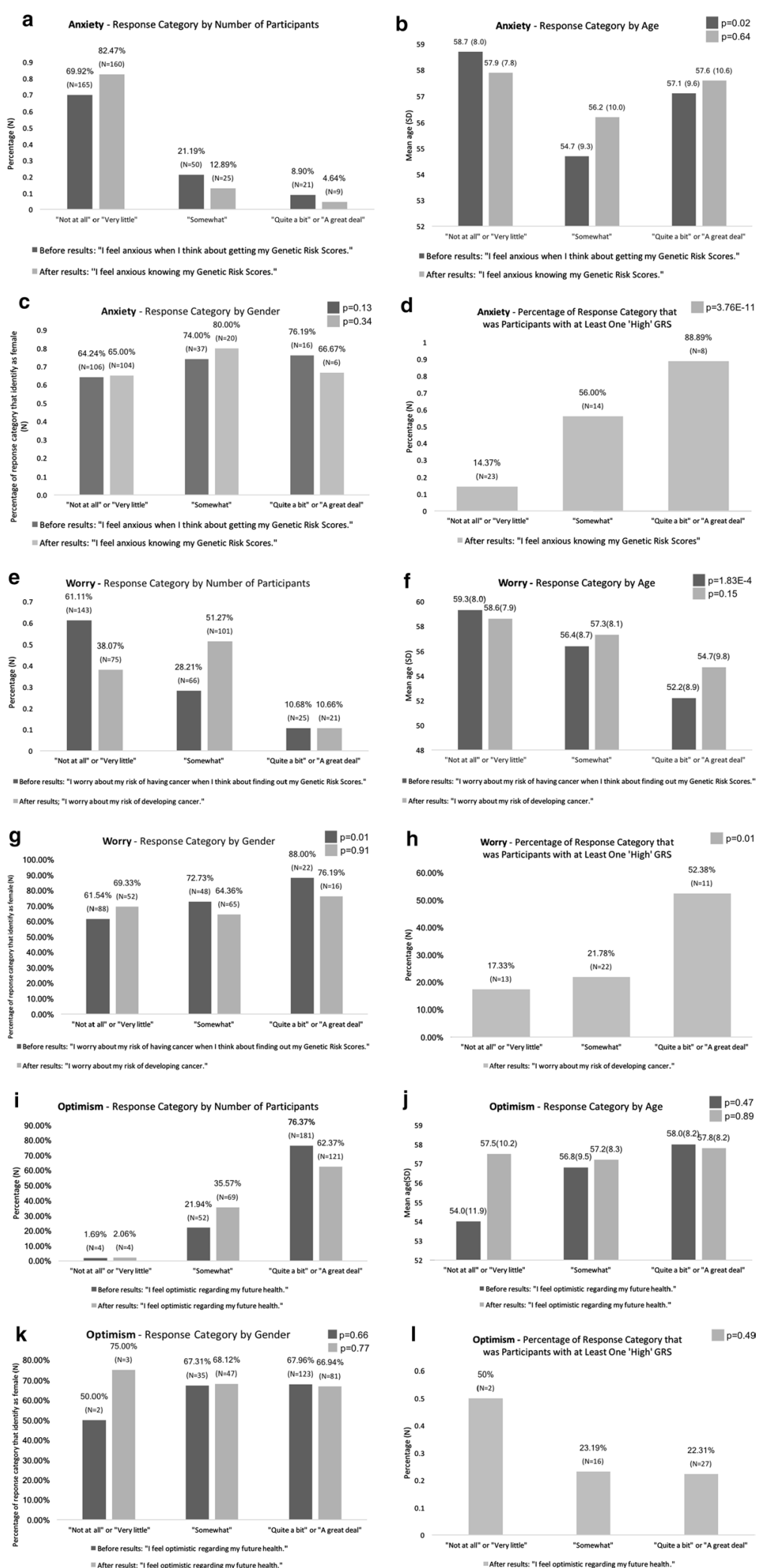

Fig. 2 Feelings regarding GRS-Related health before vs after getting GRS results 
or "very little," only $17.3 \%$ had at least one high GRS $(p=3.76 \mathrm{E}-11)$. Similarly, those who had at least one reported high GRS agreed significantly more strongly with the statement that they were worried about their risk of developing cancer ( $\mathrm{p}=0.01$ ) (Fig. 2).

There were no significant differences found between gender, age, or GRS with regards to participants' reported optimism about their future health neither before nor after receiving GRS results (Fig. 2).

\section{Self-reported cancer screening plans}

Participants were asked broadly to self-report their cancer screening plans. Before receiving GRS results, younger patients were more likely to change their screening behavior based on GRS results (Fig. 3). The average age of those agreeing most strongly was 56.4 years, whereas the average age of those somewhat agreeing was 59.5 years, and those agreeing the least as 60.1 years $(\mathrm{p}=0.01)$.

After receiving GRS results, participants responded to the statement "I plan to change my cancer screening frequency" by saying that they planned to undergo screening less frequently, with the same frequency, or more frequently (or for the first time). Of people who planned to undergo cancer screening more frequently or for the first time, significantly more had at least 1 high GRS (60\%) compared with those who planned to undergo cancer screening with the same frequency $(15.8 \%)$ or less frequently $(0 \%)(\mathrm{p}=2.7 \mathrm{E}-8)($ Fig. 3$)$.

\section{Discussion}

We conducted a clinical utility study of Genetic Risk Scores for cancer risk assessment in a primary care setting. Our goal was to provide a tool that could help further risk stratify patients to ultimately guide more personalized cancer screening plans.

Currently, though colorectal cancer screening recommendations are fairly standard and generally agreed upon by experts, risk is based primarily on family history, recommending earlier and more frequent screening (with colonoscopy) for those with a first- or second-degree family member diagnosed with colorectal cancer [4, $28,29]$. For breast cancer, guidelines regarding screening mammography initiation and frequency have been inconsistent for decades. Currently, the two major guidelines used in primary care are those of the U.S. Preventive Services Task Force (USPSTF) and American Cancer Society. While the American Cancer Society continues to recommend annual mammography screening for all healthy women beginning at age 45 , with the option to begin at age 40, the USPSTF now recommends, as of 2009 , biannual screening beginning at age 50 , unless one has a family history of breast cancer in a first-degree family member [30, 31]. For prostate cancer, a major shift in screening was made in 2012, when the large, U.S., multi-institutional Prostate, Lung, Colorectal, Ovarian (PLCO) clinical trial found prostate specific antigen (PSA) screening to be ineffective in reducing prostate cancer mortality $[32,33]$. Based on these results, the USPSTF recommended that PSA screening no longer be performed [34].

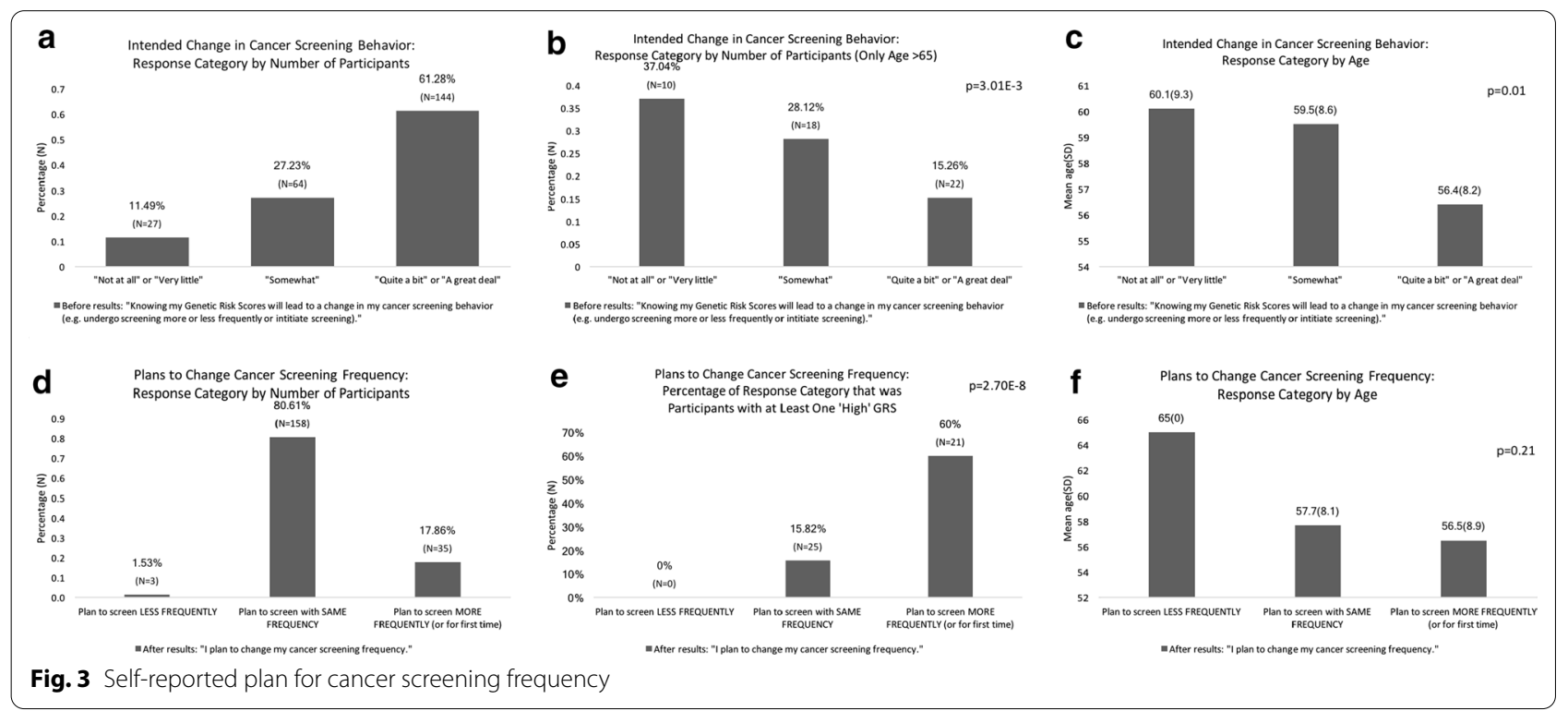


Divergent and conflicting recommendations regarding the initiation and frequency of colorectal, breast, and prostate cancer screening leave many individuals unsure about whether, when, and how often to undergo colonoscopy, screening mammography, or PSA testing. Individuals at increased risk are often advised to undergo screening earlier and more often, but these recommendations are largely based on family history, which, if known at all, may be incomplete or inaccurate. Using our GRS risk-prediction model in combination with family history, patients in a primary care setting can better understand their own individual risk for developing colorectal and either breast or prostate cancer. Afforded this new knowledge, we anticipated that patients at increased risk of developing colorectal, breast, and prostate cancer would be motivated to undergo appropriate cancer screening.

We found that most participants $(79.4 \%)$ were at low or average genetic risk of breast, prostate and colorectal cancers based on their GRS (Fig. 1d). Approximately onethird of participants, however, reported first- or seconddegree family histories of these cancers (Table 1). Though both are important to consider in assessing cancer risk and determining screening plans, we found that only $11.3 \%, 7.5 \%$, and $26.3 \%$ of participants who had a positive $\mathrm{FH}$ for breast, prostate or colorectal cancers, respectively, also had a high GRS for the respective disease (Additional file 3: Figure 1). Similarly, of participants who had a high GRS for breast, prostate or colorectal cancer, only 53.8, 41.7 , and $38.5 \%$ of participants reported family history of the disease (Additional file 3: Figure 1). This indicates that GRS may identify a new subset of the population at high risk for these cancers who would not have been identified as high-risk based on current risk-assessment criteria.

Though many participants were deemed low risk of developing one of the three tested cancers based on GRS, a significant concern with regards to the clinical utility of this test was whether participants at high, or even average, risk would suffer anxiety as a result of finding out their GRS. We found that those with a high-risk GRS did report significantly more anxiety, as well as worrying more about developing cancer. Of note, anxiety was measured based on how strongly participants agreed with a statement about having anxiety before and after receiving results rather than a verified anxiety screening questionnaire. To further address this concern, a systematic review was performed by Oliveri et al. to analyze the psychological implications of genetic testing [35]. Regarding genetic testing for cancer risk, they found that levels of anxiety and depression decreased significantly after receiving genetic testing results. However, these studies largely involved $B R C A 1$ and $B R C A 2$ testing for breast and ovarian cancers. Though potentially preventable with surgery, considering prophylactic surgery may lead to anxiety. The review also found that knowing results positively affected screening behaviors Alternatively, screening alone can lead to improved health outcomes.

Of note, this study was conducted in a group of particularly motivated patients as evidenced by their participation and follow up in a genetic study. Most had undergone breast $(88.2 \%)$ or prostate $(75.0 \%)$ cancer screening within 2 years of this study and/or colorectal cancer (53.5\%) within 5 years (data not reported). Thus, it was difficult to assess for increased compliance with recommended cancer screening via EMR data in this population. An important next step in assessing the clinical utility of GRSs should include patient populations with lower cancer screening compliance to assess whether 'high risk' scores result in more motivation to undergo cancer screening. Another improvement over the current study design would be a follow-up period greater than 3 years to assess the long-term compliance of participants with information about their genetic risk scores for various cancers.

Another limitation of this study was that, at present, most primary care providers do not regularly incorporate genetics into cancer risk assessment. This is, in part, due to the fact that genetics education targeted at primary care providers is lacking. A review of interventions that provide genetics education for primary care physicians found that receiving short-term genetics education did not necessarily lead to apparent changes in practice [36]. The authors also concluded that there are insufficient studies available to be able to inform, and thus improve, current genetics education tailored to primary care physicians. Further, the lack of established guidelines for PCPs to use in advising patients with known GRSs for the respective cancers is another hurdle for PCPs to incorporate GRSs into their daily practice.

It is encouraging, however, that larger prospective studies are being conducted to further assess the clinical utility of SNP-based risk scores in targeted cancer screening. Specifically, the ongoing WISDOM trial is comparing standard versus risk-based screening to determine onset and frequency of breast cancer screening via mammography for 100,000 women using a polygenic risk score based on 200 SNPs to stratify risk in the risk-based arm [37]. With knowledge provided by studies like this, it is promising that PCPs will be more comfortable utilizing genetics, and more specifically SNP-based risk scores, to guide their practice.

Through the present study, we were able to successfully incorporate genetic risk assessments for specific cancers, in the form of Genetic Risk Scores, into primary care practice. By educating 59 primary care physicians 
and reporting scores through existing EMR/patient portal workflow, GRSs were successfully reported to 281 patients. As data continues to become available regarding novel cancer-risk associated SNPs, associated with the cancers represented in this study as well as other cancers, we encourage further work to expand the use of Genetic Risk Scores in the primary care setting.

\section{Conclusions}

Genetic risk scores that quantify an individual's risk of developing breast, prostate and colorectal cancers as compared with a race-defined population average risk have potential clinical utility as a tool for risk stratification and to guide cancer screening in a primary care setting. To maximize their clinical utility and minimize anxiety associated with receiving a high score, we call for improved education programs for primary care providers to increase comfort surrounding the incorporation of genetic testing results into cancer screening plans, thereby empowering patients as a means of helping mitigate possible anxiety.

\section{Supplementary Information}

The online version contains supplementary material available at https://doi. org/10.1186/s12967-020-02699-w.

Additional file 1. Appendix A.

Additional file 3: Table 2. Risk-associated SNPs used for calculating Genetic Risk Scores.

Additional file 2: Table 1/Figure 1. Combined risk of GRS with FH within individuals.

\section{Abbreviations}

GWASs: Genome-wide association studies; SNPs: Single nucleotide polymorphisms; GRSs: Genetic Risk Scores; FH: Family history; PCPs: Primary care physicians; US HINTS: US Health Information National Trends Survey; USPSTF: The U.S. Preventive Services Task Force; PLCO: Prostate, Lung, Colorectal, Ovarian clinical trial; PSA: Prostate specific antigen.

\section{Acknowledgements}

We are grateful to the Ellrodt-Schweighauser, Chez and Melman families for establishing Endowed Chairs of Cancer Genomic Research and Personalized Prostate Cancer Care at NorthShore University HealthSystem in support of Drs. Xu and Helfand.

\section{Authors' contributions}

CAC analyzed and interpreted the patient data and was the main contributor to the manuscript. ZS was the primary statistician for the manuscript. WKR and EGP assisted with data acquisition and substantively revised the manuscript. RN, BTH, SLZ, CBB and JX made substantial contributions to the conception and design of the work. All authors read and approved the final manuscript.

\section{Funding}

This study was funded by the Program for Personalized Cancer Care at NorthShore University HealthSystem in Evanston, IL.

\section{Availability of data and materials}

The datasets generated and/or analyzed during the current study are not publicly available out of protection for individual patient privacy, but de-identified data may be available from the corresponding author on reasonable request.

\section{Ethics approval and consent to participate}

All participants were recruited from an existing IRB-approved genomic study, called the Genomic Health Initiative at NorthShore University HealthSystem. All participants provided written informed consent to participate in the current study.

\section{Consent for publication \\ Not applicable.}

\section{Competing interests}

The authors declare that they have no competing interests.

\section{Author details}

${ }^{1}$ University of Illinois College of Medicine, Chicago, IL, USA. ${ }^{2}$ Program for Personalized Cancer Care, NorthShore University HealthSystem, Evanston, IL, USA.

${ }^{3}$ Fudan Institute of Urology, Huashan Hospital, Fudan University, Shanghai, China.

Received: 13 October 2020 Accepted: 31 December 2020

Published online: 22 January 2021

\section{References}

1. Gail MH, Brinton LA, Byar DP, et al. Projecting individualized probabilities of developing breast cancer for white females who are being examined annually. J Natl Cancer Inst. 1989;81(24):1879-86. https://doi.org/10.1093/ jnci/81.24.1879.

2. Tyrer J, Duffy SW, Cuzick J. A breast cancer prediction model incorporating familial and personal risk factors [published correction appears in Stat Med. 2005 Jan 15;24(1):156]. Stat Med. 2004;23(7):1111-30. https://doi. org/10.1002/sim.1668.

3. US Preventive Services Task Force, Grossman DC, Curry SJ, et al. Screening for prostate cancer: US preventive services task force recommendation statement [published correction appears in JAMA. 2018 Jun 19;319(23):2443]. JAMA. 2018;319(18):1901-13. https://doi.org/10.1001/ jama.2018.3710.

4. US Preventive Services Task Force, Bibbins-Domingo K, Grossman DC, et al. Screening for colorectal cancer: US Preventive Services Task Force Recommendation Statement. JAMA. 2016;315(23):2564-75. https://doi. org/10.1001/jama.2016.5989.

5. Mavaddat N, Michailidou K, Dennis J, et al. Polygenic risk scores for prediction of breast cancer and breast cancer subtypes. Am J Hum Genet. 2019;104(1):21-34. https://doi.org/10.1016/j.ajhg.2018.11.002.

6. Giri VN, Knudsen KE, Kelly WK, et al. Role of genetic testing for inherited prostate cancer risk: Philadelphia Prostate Cancer Consensus Conference 2017. J Clin Oncol. 2018;36(4):414-24. https://doi.org/10.1200/ JCO.2017.74.1173.

7. Fantus RJ, Helfand BT. Germline genetics of prostate cancer: time to incorporate genetics into early detection tools. Clin Chem. 2019;65(1):749. https://doi.org/10.1373/clinchem.2018.286658.

8. Frampton MJ, Law P, Litchfield K, et al. Implications of polygenic risk for personalised colorectal cancer screening. Ann Oncol. 2016;27(3):429-34. https://doi.org/10.1093/annonc/mdv540.

9. Weigl K, Chang-Claude J, Knebel P, Hsu L, Hoffmeister M, Brenner H. Strongly enhanced colorectal cancer risk stratification by combining family history and genetic risk score. Clin Epidemiol. 2018;10:143-52. https:// doi.org/10.2147/CLEP.S145636.

10. Barber L, Gerke T, Markt SC, et al. Family history of breast or prostate cancer and prostate cancer risk. Clin Cancer Res. 2018;24(23):5910-7. https:// doi.org/10.1158/1078-0432.CCR-18-0370.

11. Brewer HR, Jones ME, Schoemaker MJ, Ashworth A, Swerdlow AJ. Family history and risk of breast cancer: an analysis accounting for family structure. Breast Cancer Res Treat. 2017;165(1):193-200. https://doi. org/10.1007/s10549-017-4325-2. 
12. Charlotte V, Sandro S, Rachael D. Cancer worry frequency vs. intensity and self-reported colorectal cancer screening uptake: a population-based study. J Med Screen. 2019;26(4):169-78.

13. McQueen A, Vernon S, Meissner H. Are there gender differences in colorectal cancer test use prevalence and corralates? Cancer Epidemiol Biomarkers Prev. 2006;15(4):782-91.

14. Meyer TJ, Miller ML, Metzger RL, Borkovec TD. Development and validation of the Penn State Worry questionnaire. Behav Res Ther. 1990:28:487-95

15. Choi $\mathrm{E}$, et al. Associations of perceived risk and cancer worry for colorectal cancer with screening behaviour. J Health Psychol. 2018;23(6):840-52. https://doi.org/10.1177/1359105316679721.

16. Xie Z, Wenger N, Stanton L. Risk estimation, anxiety, and breast cancer worry in women at risk for breast cancer: a single-arm trial of personalized risk communication. Psychooncology. 2019;28(11):2226-32. https:// doi.org/10.1002/pon.5211.

17. Caruso A, Vigna C, Gremigni P. The cancer worry scale revised for breast cancer genetic counseling. Cancer Nurs. 2018;41(4):311-9. https://doi. org/10.1097/NCC.0000000000000511.

18. Whitney C, Dorfman C, Shelby R. Reminders of cancer risk and pain catastrophizing: relationships with cancer worry and perceived risk in women with a first-degree relative with breast cancer. Fam Cancer. 2019;18(1):918. https://doi.org/10.1007/s10689-018-0082-6.

19. SEER Cancer Stat Facts: Female Breast Cancer. National Cancer Institute. Bethesda, MD, https://seer.cancer.gov/statfacts/html/breast.html

20. Wong MCS, Chan CH, Lin J, et al. Lower relative contribution of positive family history to colorectal cancer risk with increasing age: a systematic review and meta-analysis of 9.28 million individuals. Am J Gastroenterol. 2018;113(12):1819-27.

21. Roos VH, Mangas-Sanjuan C, Rodriguez-Girondo M, et al. Effects of family history on relative and absolute risks for colorectal cancer: a systematic review and meta-analysis. Clin Gastroenterol Hepatol. 2019;17(13):26572667.e9.

22. Thompson IM, Ankerst DP, Chi C, et al. Assessing prostate cancer risk: results from the Prostate Cancer Prevention Trial. J Natl Cancer Inst. 2006;98(8):529-34.

23. Thomas JA 2nd, Gerber L, Moreira DM, et al. Prostate cancer risk in men with prostate and breast cancer family history: results from the REDUCE study (R1). J Intern Med. 2012;272:85-92.

24. Chen YC, Page JH, Chen R, Giovannucci E. Family history of prostate and breast cancer and the risk of prostate cancer in the PSA era. Prostate. 2008:68(14):1582-91.

25. Ahn J, Moslehi R, Weinstein SJ, Snyder K, Virtamo J, Albanes D. Family history of prostate cancer and prostate cancer risk in the AlphaTocopherol, Beta-Carotene Cancer Prevention (ATBC) Study. Int J Cancer 2008;123(5):1154-9.

26. Ahern TP, Sprague BL, Bissell MCS, et al. Family history of breast cancer breast density, and breast cancer risk in a U.S. Breast cancer screening population. Cancer Epidemiol Biomarkers Prev. 2017;26(6):938-44.
27. R Core Team. R: slanguage and environment for statistical computing R Foundation for Statistical Computing, Vienna, Austria; 2020. http:// www.R-project.org/.

28. Wolf AMD, Fontham ETH, Church TR, et al. Colorectal cancer screening for average-risk adults: 2018 guideline update from the American Cancer Society. CA Cancer J Clin. 2018;68(4):250-81. https://doi.org/10.3322/ caac. 21457

29. Provenzale D, Gupta S, Ahnen DJ, et al. NCCN guidelines insights: colorectal cancer screening Version. J Natl Compr Canc Netw. 2018;16(8):939-49. https://doi.org/10.6004/jnccn.2018.0067.

30. Siu AL, U.S. Preventive Services Task Force. Screening for Breast Cancer: US Preventive Services Task Force Recommendation Statement [published correction appears in Ann Intern Med. 2016 Mar 15;164(6):448]. Ann Intern Med. 2016;164(4):279-96. https://doi.org/10.7326/M15-2886.

31. Oeffinger KC, Fontham ET, Etzioni R, et al. Breast cancer screening for women at average risk: 2015 guideline update from the American Cancer Society [published correction appears in JAMA. 2016 Apr 5;315(13):1406]. JAMA. 2015;314(15):1599-614. https://doi.org/10.1001/jama.2015.12783.

32. Pinsky PF, Black A, Parnes HL, et al. Prostate cancer specific survival in the Prostate, Lung, Colorectal, and Ovarian (PLCO) cancer screening trial. Cancer Epidemiol. 2012;36(6):e401-6. https://doi.org/10.1016/j.canep 2012.08.008

33. Andriole GL, Crawford ED, Grubb RL 3rd, et al. Prostate cancer screening in the randomized prostate, lung, colorectal, and ovarian cancer screening trial: mortality results after 13 years of follow-up. J Natl Cancer Inst. 2012;104(2):125-32. https://doi.org/10.1093/jnci/djr500.

34. Moyer VA, U.S. Preventive Services Task Force. Screening for prostate cancer: U.S. Preventive Services Task Force recommendation statement. Ann Intern Med. 2012;157(2):120-34. https://doi.org/10.7326/0003-4819157-2-201207170-00459.

35. Oliveri S, Ferrari F, Manfrinati A, Pravettoni G. A systematic review of the psychological implications of genetic testing: a comparative analysis among cardiovascular, neurodegenerative and cancer diseases. Front Genet. 2018;9:624. https://doi.org/10.3389/fgene.2018.00624.

36. Paneque $M$, Turchetti $D$, Jackson L, Lunt $P$, Houwink $E$, Skirton $H$. A systematic review of interventions to provide genetics education for primary care. BMC Fam Pract. 2016;17:89. https://doi.org/10.1186/s1287 5-016-0483-2.

37. Esserman LJ, WISDOM Study and Athena Investigators. The WISDOM Study: breaking the deadlock in the breast cancer screening debate. NPJ Breast Cancer. 2017;3:34. https://doi.org/10.1038/s41523-017-0035-5.

\section{Publisher's Note}

Springer Nature remains neutral with regard to jurisdictional claims in published maps and institutional affiliations.
Ready to submit your research? Choose BMC and benefit from:

- fast, convenient online submission

- thorough peer review by experienced researchers in your field

- rapid publication on acceptance

- support for research data, including large and complex data types

- gold Open Access which fosters wider collaboration and increased citations

- maximum visibility for your research: over 100M website views per year

At BMC, research is always in progress.

Learn more biomedcentral.com/submissions 